\title{
3D Distributed Memory Polynomial Behavioral Model for Concurrent Dual-Band Envelope Tracking Power Amplifier Linearization
}

\author{
Pere L. Gilabert, Senior Member, IEEE, and Gabriel Montoro, Member, IEEE
}

\begin{abstract}
This paper presents a new three-dimensional (3D) behavioral model to compensate for the nonlinear distortion arising in concurrent dual-band (DB) Envelope Tracking (ET) Power Amplifiers (PAs). The advantage of the proposed 3D distributed memory polynomial (3D-DMP) behavioral model, in comparison to the already published behavioral models used for concurrent dual-band envelope tracking PA linearization, is that it requires a smaller number of coefficients to achieve the same linearity performance, which reduces the overall identification and adaptation computational complexity. The proposed 3DDMP digital predistorter (DPD) is tested under different ET supply modulation techniques. Moreover, further model order reduction of the 3D-DMP DPD is achieved by applying the principal component analysis (PCA) technique. Experimental results are shown considering a concurrent $D B$ transmission of a WCDMA signal at $1.75 \mathrm{GHz}$ and a $10 \mathrm{MHz}$ bandwidth LTE signal at 2.1 GHz. The performance of the proposed 3D-DMP DPD is evaluated in terms of linearity, drain power efficiency and computational complexity.
\end{abstract}

Index Terms-digital predistorter, dual-band, envelope tracking, order reduction, RF power amplifiers, principal component analysis.

\section{INTRODUCTION}

$\mathbf{S}$ EVERAL efforts have been dedicated in recent past years to design wireless communication systems capable to deal with multi-standard or multi-band signals at the same time. In the specific case of dual-band (DB) systems, intensive research has been devoted to design single PAs to concurrently support dual frequency bands with significant frequency separation, that is, from several hundreds of $\mathrm{MHz}$ up to few $\mathrm{GHz}$ [1] [4]. The advantage of having one single power amplifier (PA) able to process signals in multiple bands simultaneously is the reduction of the number of components and cost of the RF subsystem.

Nevertheless, the PA has to cope with spectrally efficient modulated signals presenting wide bandwidth (BW) and high peak-to-average power ratio (PAPR). Signals presenting high

This work was partially supported by the Spanish Government (MINECO) under project TEC2011-29126-C03-02; by the Catalan Government (Secretaria d'Universitats i Recerca del Departament d'Economia i Coneixement de la Generalitat de Catalunya) under project 2014-SGR-1103; and by the Cluster for Application and Technology Research in Europe on Nanoelectronics (CATRENE) under the project named CORTIF CA116 - Coexistence of Radio Frequency Transmission in the Future.

Pere L. Gilabert and Gabriel Montoro are with the Department of Signal Theory and Communications, Universitat Politècnica de Catalunya (UPC) Barcelona Tech. C/ Esteve Terradas, 7, 08860 Castelldefels, Barcelona, Spain. (+34 934137 211; e-mail: plgilabert@tsc.upc.edu, gabriel.montoro@upc.edu)

Manuscript received September XX, 2014; revised XXXX XX, 2014.
PAPR have a negative impact in the transmitter's power efficiency, because the PA has to be operated at high backoff levels to avoid introducing nonlinear distortion. Even by compensating for the nonlinear distortion using digital predistortion (DPD) [5], the power efficiency improvement that can be achieved is limited. To significantly improve the power efficiency figures, dynamic supply modulation techniques, such as envelope tracking (ET) [6], have been proposed in dual-band systems [7]-[11].

The combination of concurrent dual-band PAs with envelope tracking faces several challenges. On the one hand, to guarantee the desired linearity levels, the DPD has to be designed taking into account the difficulty of running the DPD at around $5 \mathrm{x}$ the signal's BW due to the BW expansion occurring in the DPD process. When considering bands separated several hundreds of $\mathrm{MHz}$, the implementation of a wideband DPD is unfeasible, especially in real-time platforms. Fortunately, DPD systems for dual-band signals can be significantly simplified assuming that the nonlinear distortions of concern are those that arise close to the band of interest, while the rest could be removed by filtering. This is the idea behind the twodimensional (2D) behavioral models presented in [12]-[17].

On the other hand, one of the main challenges in envelope tracking for concurrent dual-band regards the design of efficient envelope drivers capable of supplying the power required by the transistor at the same speed of the signal's envelope. In a concurrent DB transmission, the envelope of the resulting RF signal can present BWs that are several times the carrier separation (according to the rule of thumb around $3 \mathrm{x}$ ). In order to avoid dealing with this high speed envelope variations (normally, the envelope driver's efficiency decays with the envelope's BW) some methods to reduce the BW [18] or slewrate (SR) [19] of the signal's envelope have been proposed.

Alternatively, for concurrent dual-band signals, there are at least two methods to deal with the instantaneous dual-band envelope of the transmitted signal: a) perform the sum of the modulus of the baseband signals (i.e., the peak of the instantaneous dual-band envelope), as proposed by Gilabert et al. in [8]; or b) perform the square root of the sum of the squared modulus of the baseband signals (i.e., the average amplitude of the instantaneous dual-band envelope), as proposed by Lin et al. in [9].

Unfortunately, using a different envelope (i.e., intentionally slower) than the original instantaneous dual-band envelope to dynamically supply the PA produces additional nonlinear distortion. As a consequence, in order to compensate later for 


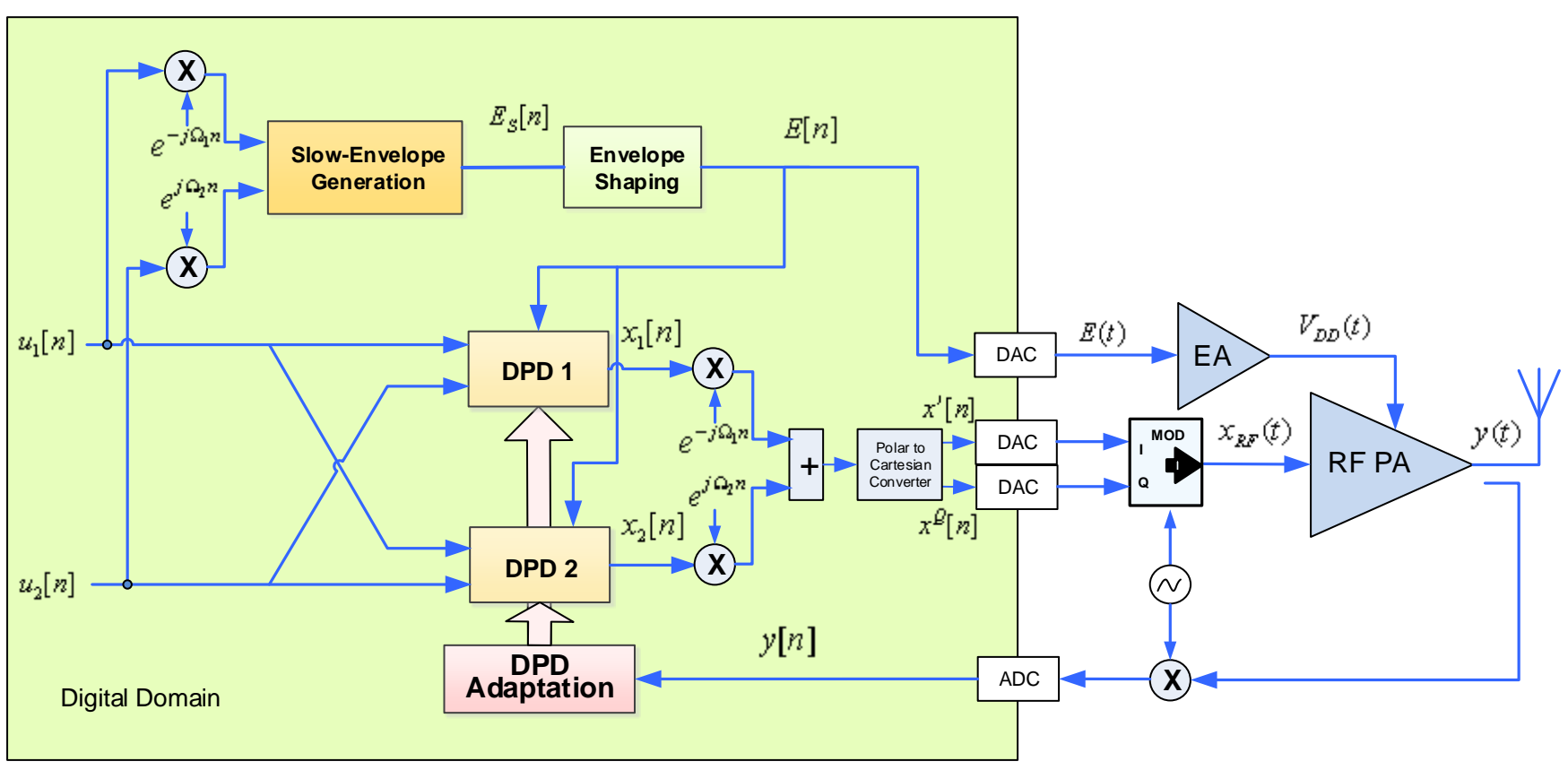

Fig. 1. Block diagram of the 3D DPD and slow envelope generator for concurrent dual-band envelope tracking PAs.

the slow-envelope dependent distortion effects, a concurrent dual-band envelope tracking PA behavioral model has to include in its formulation not only the interference band signal, but also the envelope used for dynamically supply the PA. So, it results in a three-dimensional (3D) behavioral model as described in [8]-[11].

The number of coefficients required by these 3D behavioral models grows dramatically when considering memory effects. This has a negative impact in the least squares (LS) based model extraction because it increases the computational complexity and can lead to a bad conditioning extraction. Several efforts have been made to solve the ill-conditioning problem as well as to reduce the model extraction errors when using a small number of data samples to characterize PA behavioral models [20]. Alternatively, reducing the order of the DPD model by properly selecting the most significant basis functions or creating a new set of orthogonal basis functions, has beneficial effects in both the computational complexity and in the conditioning of the data matrices. The singular value decomposition (SVD) [21]-[24] or the principal component analysis (PCA) technique [25] are commonly used for extracting the dominant eigenvalues/eigenvectors and thus reducing the order of the DPD function. By creating a new orthogonal basis it is possible to reduce the order of the DPD behavioral model (and at the same time improve the matrix conditioning) where, unlike other structured or intelligent pruning approaches, the model order reduction is made without assuming any specific physical structure of the model.

This paper presents a new 3D behavioural model that significantly reduces the computational complexity of the DPD function. It is based on a 3D distributed memory polynomial (3DDMP) architecture in which, following a parallel structure, each branch is responsible for characterizing/compensating one of the three main (intra-band, cross-band and dynamic supply distortion) unwanted nonlinear distortion effects in concurrent dual-band envelope tracking PAs. The linearity performance (quantized in terms of ACLR and NMSE) and computational complexity (quantized in terms of number of coefficients) of the proposed 3D-DMP DPD is compared, to the best authors knowledge, to the most recent published 3D behavioral models for linearizing concurrent dual-band envelope tracking PAs. These models, sorted according to its publication date, are: the 3D memory polynomial (3D-MP) model proposed by Gilabert et al. in [8], the 3D baseband equivalent Volterra series (3D-BBE Volterra) model proposed by Sarbishaei et al. in [10] and the 2D phase-aligned Volterra series (2D P-A Volterra) model proposed by Kwan et al. in [11]. Moreover, the 3D-DMP DPD is tested for different envelope tracking supply modulation strategies to evaluate the linearity and the drain efficiency performance of the dual-band envelope tracking PA system, schematically depicted in Fig. 1. Finally, to further reduce the computational complexity of the DPD, a model order reduction technique following the PCA theory is also applied.

Therefore, the paper is organized as follows. In Section II, we analyze different strategies to generate a slow envelope to dynamically supply concurrent dual-band PAs. Then, in Section III we present and compare the proposed 3D-DMP behavioral model to other already published solutions in terms of required number of coefficients according to the specific architecture of each model. Section IV is devoted to describe the experimental setup. In Section $\mathrm{V}$, the linearization, power and computational efficiency performance of the proposed 3DDMP DPD is evaluated under different envelope tracking supply modulation strategies and compared to the aforementioned 3D-DPD models. Finally, conclusions are given in Section VI. 


\section{Supply Modulation Techniques fOR CONCURRENT DUAL-BAND AMPLIFICATION}

In an envelope tracking system, the supply voltage is dynamically adjusted to follow the instantaneous RF envelope. When considering concurrent dual-band operation, where the instantaneous dual-band envelope presents a BW (according to the rule of thumb) around $3 \mathrm{x}$ the carrier separation, it is mandatory to use a slower version of the original envelope to relax the envelope driver's BW and SR requirements. However, the price for not using the original RF envelope to supply the PA is the degradation of the power efficiency and linearity.

In this paper we will evaluate the linearization performance of the proposed 3D-DMP under different supply modulation strategies used to generate a slower envelope capable to meet the envelope driver's limitations. Moreover, a comparison of the drain power efficiency obtained with these different ET supply modulation techniques will be also presented. In particular, the following strategies will be considered:

- Dynamic supply based on the generalized mean (GM) of the instantaneous dual-band envelope.

- Dynamic supply based on the combination of both slewrate (SR) and bandwidth (BW) reduced (SR\&BWred) envelopes of the instantaneous DB envelope.

We can define the generalized mean (also known as power mean or Hölder mean) with exponent $p$ (with $p$ being a nonzero real number) of the positive real numbers $x_{1}, \ldots, x_{K}$ as

$$
G M_{p}=\left(\frac{1}{K} \sum_{i=1}^{K} x_{i}^{p}\right)^{1 / p}
$$

where for $p=1$ we obtain the arithmetic mean and for $p=2$ the root mean square. Therefore, the envelope tracking supply modulation technique based on the generalized mean (considering two sequences) is described in the following equation,

$$
E_{s}^{G M_{p}}[n]=\left(\frac{1}{2}\left(\left|u_{1}[n]\right|^{p}+\left|u_{2}[n]\right|^{p}\right)\right)^{1 / p}
$$

where $u_{1}[n]$ and $u_{2}[n]$ are the input discrete-time complex baseband signals, as shown in Fig. 1, and with the exponent $p$ being a non-zero real number. In concrete we will evaluate the GM for the following values of $p$ :

- GM with $p=1$ : Peak of the instantaneous DB envelope [8].

- GM with $p=2$ : Average of the instantaneous DB envelope [9].

- GM with $p=1.5$.

- GM with $p=4$.

The reason for choosing also $p=1.5$ and $p=4$ is to observe the shape of the resulting slow envelope in both time and frequency domain and determine if it has a significant effect on the linearity and efficiency of the overall dual-band envelope tracking system.

On the other hand, the envelope tracking supply modulation technique based on the SR\&BWred version of the instantaneous dual-band envelope is defined as

$$
E_{s}^{S R \& B W}[n]=\min \left\{E_{s}^{G M_{1}}[n], E_{s}^{S R}[n]\right\}
$$
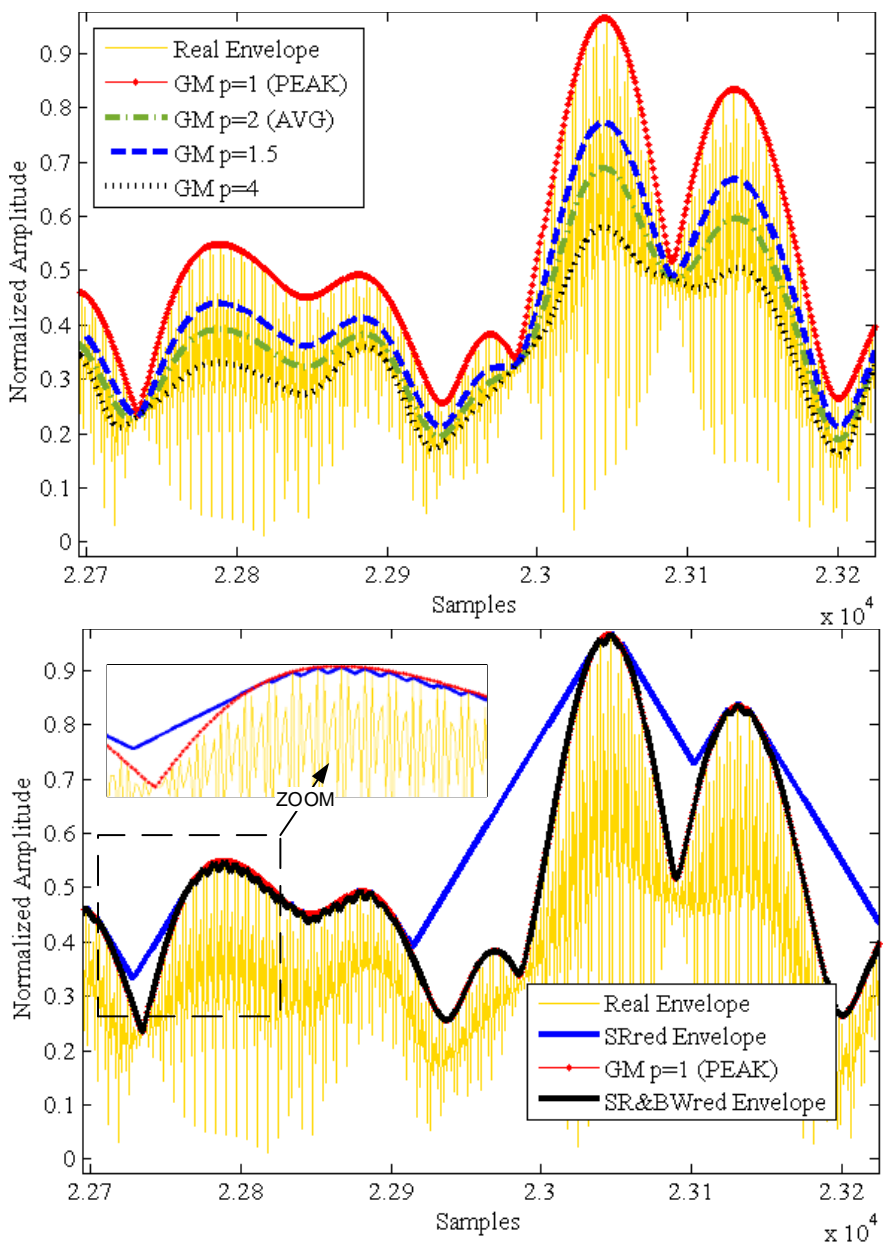

Fig. 2. Waveforms of envelopes for dual-band envelope tracking PAs.

where $E_{s}^{G M_{1}}$ is the slow envelope based on the peak of the instantaneous dual-band envelope and where $E_{s}^{S R}[n]$ is the SR reduced version of the original dual-band instantaneous envelope that can be defined as

$$
E_{s}^{S R}[n]=\Gamma\left(\left|u_{1}[n] e^{-j \Omega_{1} n}+u_{2}[n] e^{j \Omega_{2} n}\right|\right)
$$

where $\Gamma(\cdot)$ is the SR reduction transformation extensively described in [19], [26]. With the SR reduction algorithm we can limit the maximum slew-rate of the resulting envelope. However, the resulting slew-rate reduced envelope is not bandlimited, mainly due to the presence of peaks. These peaks are introduced by the algorithm when it is necessary to change drastically from a positive to a negative slope, or vice-versa.

This SR\&BWred technique tries to maximize the drain efficiency by reducing both the SR and BW of the original instantaneous dual-band envelope at the limit supported by a specific envelope driver.

Fig. 2 and Fig. 3 show the time-domain waveforms and spectra, respectively, of the instantaneous dual-band envelope (considering a WCDMA signal at $1.75 \mathrm{GHz}$ and a $10 \mathrm{MHz}$ BW LTE signal at $2.1 \mathrm{GHz}$ ) and the proposed slow envelopes based on both the GM and the SR\&BWred techniques. Regarding the slow envelopes generated considering the GM approach, we can observe that for $p=1$ we obtain (see 

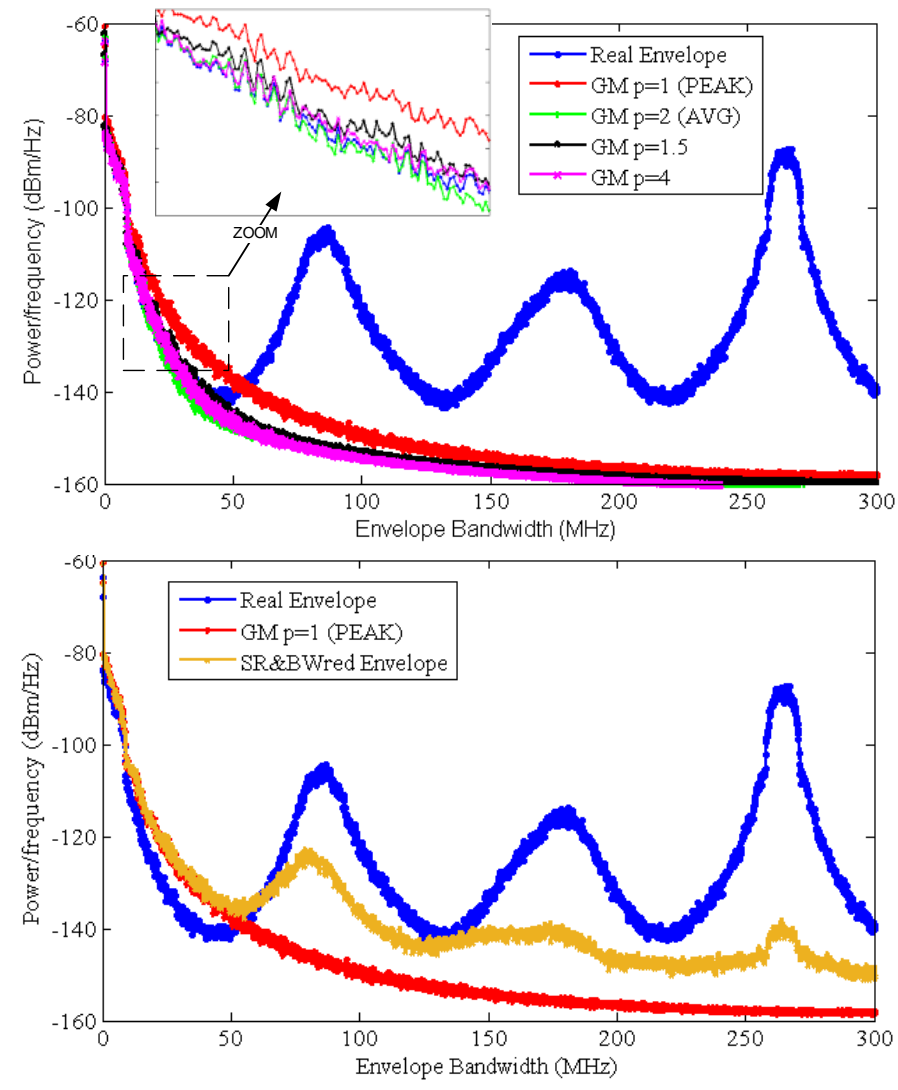

Fig. 3. Spectra of envelopes for dual-band envelope tracking PAs.

Fig. 2-top) an upper bound (peak envelope) of the original $\mathrm{RF}$ envelope (Minkowski inequality),

$$
\left|u_{1}[n] e^{-j \Omega_{1} n}+u_{2}[n] e^{j \Omega_{2} n}\right| \leq\left|u_{1}[n]\right|+\left|u_{2}[n]\right|
$$

and its BW (Fig. 3-top) is significantly smaller than the original one. However, the narrowest envelope BW is obtained with $p=2$ (average envelope). As it will be shown later, no significant differences in terms of linearity and drain efficiency are obtained by considering alternative values (e.g., $p=1.5$ or $p=4)$. In addition, when $p$ tends to 0 the slow envelope waveform tends to a constant supply voltage value different than 0 (for example 1 in the case of having normalized waveforms), while if $p$ tends to infinity the resulting slow envelope tends to 0 .

On the other hand, Fig. 2-bottom shows the resulting SR\&BWred envelope considering a SR reduction of $98.87 \%$ with respect to the original instantaneous dual-band envelope. The slew-rate reduction (SRred) percentage is defined as

$$
\operatorname{SRred}(\%)=\frac{S R_{O}-S R_{S}}{S R_{O}} \cdot 100
$$

where $S R_{O}$ is the slew-rate of the instantaneous dual-band envelope and $S R_{S}$ is the slew-rate of the resulting envelope after applying the slew-rate reduction algorithm. As it can be observed in Fig. 3-bottom, the resulting BW still presents significant information at high frequency values (when compared to $G M_{1}$, for example), this is the price to pay in order to try to maximize the drain efficiency. In general, despite the efficiency

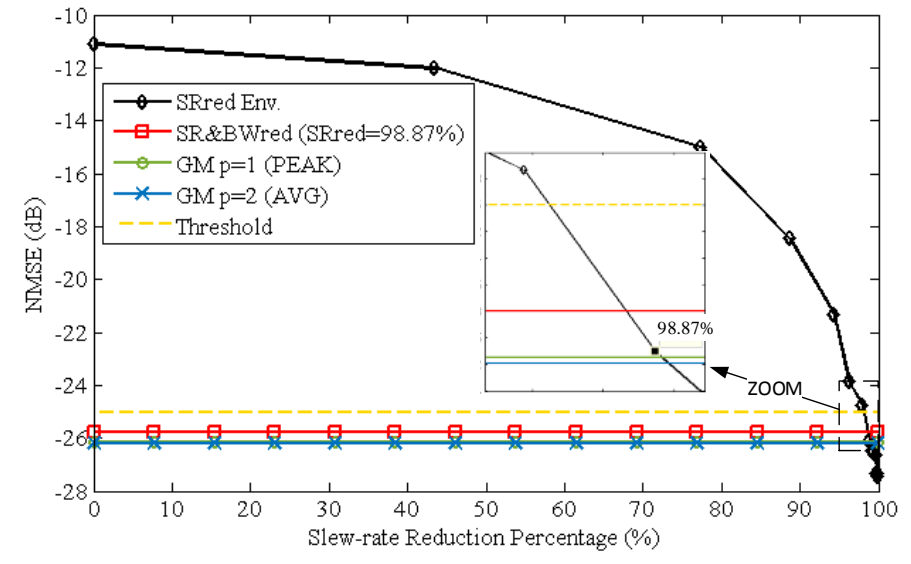

Fig. 4. Measured NMSE between the input and output supply envelope at the EA for different supply modulation techniques and SR reduction percentages.

of the supply modulator can even be improved by using a slower envelope (bandwidth or slew-rate limited envelope), the overall drain efficiency will be degraded in comparison to the efficiency obtained using the original envelope [18].

To empirically determine which is the maximum SR or envelope BW supported by a specific envelope driver, we can evaluate the normalized mean square error (NMSE) between the input and output signals at the envelope driver. In our particular case (the envelope driver will be later described in the Section IV), we have noticed that defining a threshold of $-25 \mathrm{~dB}$ of NMSE as the minimum allowed reliability between input and output signals at the envelope driver, we can later guarantee the linearity levels of the RF output dual-band signal using the proposed 3D-DMP DPD. Fig. 4 shows the NMSE when using SR reduced envelopes with different $\mathrm{SR}$ reduction percentages and also for the peak $\left(G M_{1}\right)$, average $\left(G M_{2}\right)$ and SR\&BWred envelopes. As observed in Fig. 4, in our particular case, the minimum SR reduction percentage that already meets the $-25 \mathrm{~dB}$ of NMSE is around $98.5 \%$. Consequently, the SR\&BWred envelope has been generated using a similar SR reduction percentage.

Finally, as depicted in Fig. 1, the generated slow envelope, taking into account one of the aforementioned techniques, is then shaped according to the shaping function described in [1] with the objective of maximizing power efficiency,

$$
E[n]=\left(\left(E_{s \_T H}\right)^{6}+\left(E_{s}^{x x}[n]\right)^{6}\right)^{1 / 6}
$$

where $E_{s_{-} T H}$ is the lower bound of the envelope that determines the swing voltages or dynamic range of the supply modulator (normally set to avoid the knee region unwanted effect) and where $E_{s}^{x x}[n]$ can be either $E_{s}^{G M_{p}}[n]$ or $E_{s}^{S R \& B W}[n]$, depending on the strategy selected to generate the slow envelope. We now define the lower threshold percentage (LTP) of the supply envelope voltage as

$$
\operatorname{LTP}(\%)=\frac{E_{s_{-} T H}}{\max \left(E_{s}^{x x}[n]\right)} \cdot 100
$$

As expected, the higher the LTP, the better the overall linearity and the worse the drain efficiency, which at the limit would correspond to constant supply (i.e., $L T P=100 \%$ ). 


\section{3D Distributed Memory Polynomial Digital PREDISTORTER}

\section{A. Antecedents}

In order to take into account the slow-envelope dependent distortion effects that appear in the dynamic supply of the PA, the concurrent dual-band envelope tracking PA behavioral model has to include in its formulation not only the interference band signal, but also the envelope used for dynamically supply the PA. Therefore, it results a 3D behavioral model,

$$
\begin{aligned}
& \hat{y}_{1}[n]=f_{1}\left(x_{1}[n], x_{2}[n], E[n]\right) \\
& \hat{y}_{2}[n]=f_{2}\left(x_{2}[n], x_{1}[n], E[n]\right)
\end{aligned}
$$

where $\hat{y}_{1}[n]$ and $\hat{y}_{2}[n]$ are the estimated complex baseband outputs of the 3D PA behavioral model at each frequency band, respectively. These behavioral models are estimated from complex input and output data records (see Fig. 1), where

$$
y[n]=y_{1}[n] e^{-j \Omega_{1} n}+y_{2}[n] e^{j \Omega_{2} n}
$$

To properly characterize and later compensate for the the inband and out-of-band intermodulation, cross-band modulation distortion and the slow-envelope dependent distortion effects that appear in concurrent dual-band transmitters with dynamic supply, a 3D memory polynomial (3D-MP) DPD was proposed by the authors in [8]. Following the notation in Fig. 1, the DPD output (for Band 1) was defined as

$$
\begin{array}{r}
x_{1}[n]=\sum_{i=0}^{N-1} \sum_{q=0}^{Q-1} \sum_{p=0}^{P-1} \psi_{p q i}^{(1)}(E) \cdot u_{1}\left[n-\tau_{i}^{u}\right] \\
\left|u_{1}\left[n-\tau_{i}^{u}\right]\right|^{p}\left|u_{2}\left[n-\tau_{i}^{u}\right]\right|^{q}
\end{array}
$$

where $u_{1}[n]$ and $u_{2}[n]$ are complex baseband signals to be transmitted which, as extensively explained by Roblin et al. in [27], are necessary to characterize the inter-band and cross-band intermodulation distortion in concurrent dual-band PAs. In addition, to properly characterize the slow-envelope distortion effects that appear when supplying the PA with a slower version of the instantaneous dual-band envelope, the coefficients $\psi_{p q i}^{(1)}(E)$ are dependent on the slow envelope $(E[n])$ and its memory,

$$
\psi_{\text {pqi }}^{(1)}(E)=\sum_{m=0}^{M-1} \sum_{r=0}^{R-1} w_{\text {pqirm }}^{(1)} \cdot\left(E\left[n-\tau_{m}^{e}\right]\right)^{r}
$$

where $w_{\text {pqirm }}^{(1)}$ are the complex coefficients describing the model, $\tau^{u}$ and $\tau^{e}$ (with $\tau^{u, e} \in \mathbb{Z}$ and $\tau_{0}^{u, e}=0$ ) are the most significant sparse delays of the input $\left(u_{1}[n]\right)$, interference signal $\left(u_{2}[n]\right)$ and slow envelope $(E[n])$ that contribute to characterize memory effects and time misalignments between the supply voltage waveform and the RF modulated signal. Analogously, $x_{2}[n]$ can be described as in (11) and (12) but considering $u_{2}[n]$ as the input signal and $u_{1}[n]$ as the interfering one, while $w_{\text {pqirm }}^{(2)}$ are the parameters describing the DPD in Band 2.

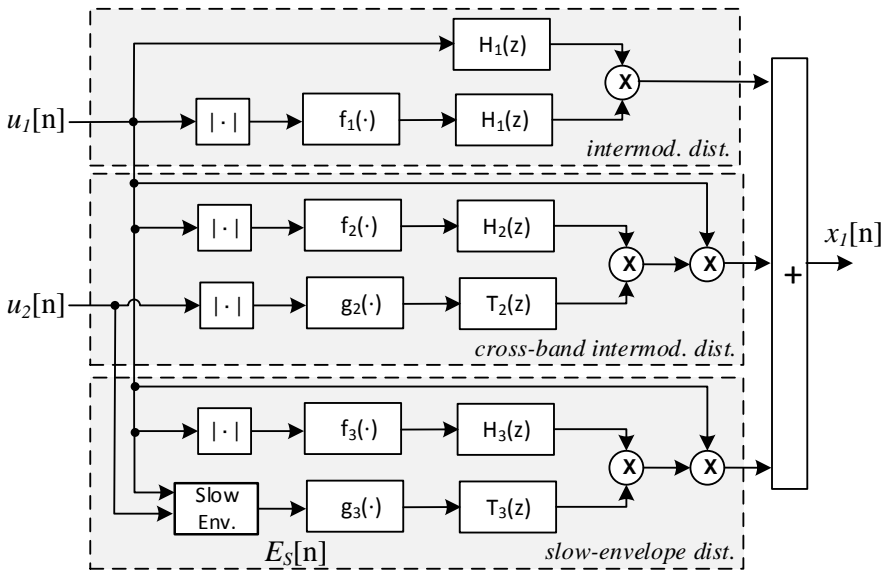

Fig. 5. Structure of the proposed 3D-DMP DPD for Band 1.

\section{B. Description of the New 3D Distributed Memory Polynomial Digital Predistorter}

One of the problems of the 3D-MP DPD in [8] is the potential growth of the number of coefficients required when considering memory effects in both input signals or the slow envelope. This is an issue not only because of the computational complexity introduced by the DPD, but also because dealing with a huge number of coefficients may take to an ill-conditioned identification due to over-parametrization and lack of orthogonality among the considered basis functions. As a consequence, several efforts have been devoted to find simplified structures. This is the case of the multi-dimensional Volterra-based models such as the 3D-BBE Volterra model [10] or 2D P-A Volterra model [11]. Alternatively, or in a complementary way, model order reduction techniques such as the ones based on the SVD [21]-[23] or techniques based on the PCA theory [28] are applied without assuming any $a$ priori physical structure of the model.

In this paper we propose a new 3D distributed memory polynomial (3D-DMP) DPD, as schematically depicted in Fig. 5. The objective of this new 3D DPD is to reduce the required number of parameters to compensate for the nonlinear distortion in concurrent dual-band envelope tracking PAs. This is possible by following a distributed structure. The strategy of adding several structures in parallel fosters the identification accuracy when considering linearly independent basis functions and significantly reduces the number of coefficients with respect to using a series approach, such as in the 3D-MP DPD in [8], where all the unwanted effects were considered to be inter-related. Therefore, the main advantage of this distributed model regards the possibility of integrating several structures in parallel, each one aiming at compensating a specific nonlinear distortion, and thus providing flexibility in the design.

The proposed 3D-DMP DPD includes three branches to compensate for in-band, out-of-band and cross-band intermodulation distortion and the slow-envelope distortion, respectively. A memoryless representation of the proposed model 
for Band 1 is

$$
\begin{array}{r}
x_{1}[n]=u_{1}[n] \cdot\left(f_{1}\left(\left|u_{1}[n]\right|\right)+\right. \\
\left.\beta\left(\left|u_{2}[n]\right|\right) \cdot f_{2}\left(\left|u_{1}[n]\right|\right)+\gamma(E[n]) \cdot f_{3}\left(\left|u_{1}[n]\right|\right)\right)
\end{array}
$$

If we now take into account a model with memory, the inputoutput relationship in the 3D-DMP DPD (for Band 1) is defined as

$$
\begin{array}{r}
x_{1}[n]=\sum_{i=0}^{N_{1}-1} \sum_{p=0}^{P_{1}-1} a_{p i}^{(1)} \cdot u_{1}\left[n-\tau_{i}^{u_{1}}\right]\left|u_{1}\left[n-\tau_{i}^{u_{1}}\right]\right|^{p}+ \\
\sum_{i=1}^{N_{2}-1} \sum_{p=0}^{P_{2}-1} \beta_{p i}^{(1)}\left(u_{2}\right) \cdot u_{1}[n]\left|u_{1}\left[n-\tau_{i}^{u_{1}}\right]\right|^{p}+ \\
\sum_{i=1}^{N_{3}-1} \sum_{p=0}^{P_{3}-1} \gamma_{p i}^{(1)}(E) \cdot u_{1}[n]\left|u_{1}\left[n-\tau_{i}^{u_{1}}\right]\right|^{p}
\end{array}
$$

where coefficients $\beta_{p i}^{(1)}\left(u_{2}\right)$ are dependent on the interference signal $\left(u_{2}[n]\right)$ and its memory,

$$
\beta_{p i}^{(1)}\left(u_{2}\right)=\sum_{j=1}^{M_{2}-1} \sum_{q=0}^{Q_{2}-1} b_{p i q j}^{(1)}\left|u_{2}\left[n-\tau_{j}^{u_{2}}\right]\right|^{q}
$$

and coefficients $\gamma_{p i}^{(1)}(E)$ are dependent on the slow-envelope $(E[n])$ and its memory.

$$
\gamma_{p i}^{(1)}(E)=\sum_{k=1}^{K_{3}-1} \sum_{r=0}^{R_{3}-1} c_{p i r k}^{(1)}\left(E\left[n-\tau_{k}^{e}\right]\right)^{r}
$$

Moreover, $P_{1}, P_{2}$ and $P_{3}$, are the polynomial orders of the input signal $\left(u_{1}[n]\right)$ at each branch; $N_{1}, N_{2}$ and $N_{3}$, are the number of delays of the input signal at each branch; $Q_{2}$ is the polynomial order of the interference signal $\left(u_{2}[n]\right) ; M_{2}$ is the number of delays of the interference signal; $R_{3}$ the polynomial order of the supply envelope $(E[n])$ and $K_{3}$ the number of delays of the supply envelope; $\tau^{u_{1}}, \tau^{u_{2}}$ and $\tau^{e}$ (with $\tau^{u_{1}, u_{2}, e} \in \mathbb{Z}$ and $\tau_{0}^{u_{1}, u_{2}, e}=0$ ) are the most significant sparse delays of the input $\left(u_{1}[n]\right)$, interference signal $\left(u_{2}[n]\right)$ and slow envelope $(E[n])$ that contribute to characterize memory effects and time misalignments between the supply voltage waveform and the RF modulated signal.

Analogously, $x_{2}[n]$ can be described as in (14)-(16) but now considering $u_{2}[n]$ as the input signal and $u_{1}[n]$ as the interfering one.

To show the benefits of proposed 3D-DMP DPD in terms of computational complexity reduction, Fig. 6 compares the required number of coefficients of the following behavioral models: a) 3D-MP; b) 3D-DMP; c) 3D-BBE Volterra; and d) 2D P-A Volterra, when considering different memory lengths in both input/interference signals and the slow envelope. Therefore, considering different memory taps in delay $\mathrm{T} 1$ and delay T2 it is possible to evaluate the models' complexity growth in terms of number of coefficients. The correspondence of delay T1 and delay T2 in Fig. 6 with the specific parameters (following the original papers' notation) of the aforementioned models under comparison is described in the following:

- Delay T1: $N$ (3D-MP); $N_{1}$ (3D-DMP); $M_{1}$ (3D-BBE Volterra); $M_{1}$ (2D P-A Volterra).

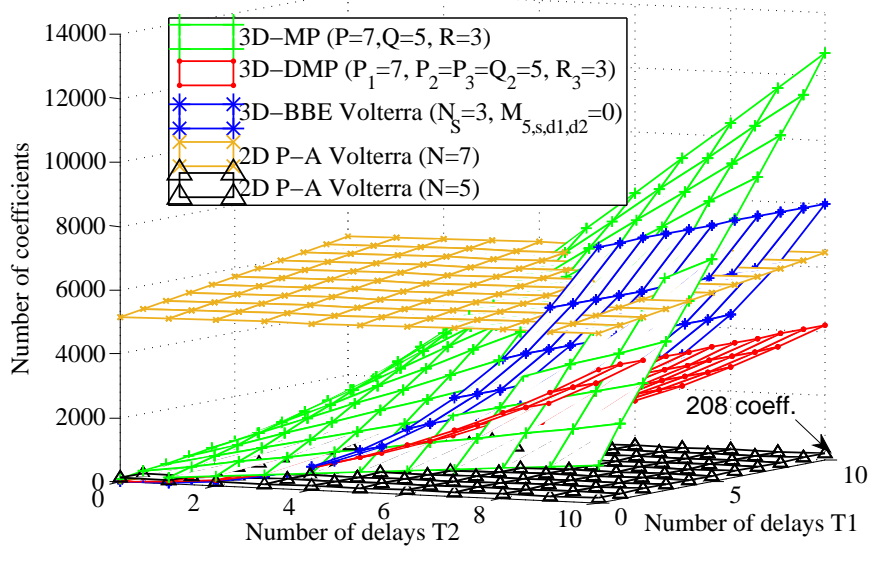

Fig. 6. Number of coefficients versus number of memory taps for different dual-band envelope tracking DPD models.

- Delay T2: $M$ (3D-MP); $N_{2}=N_{3}=M_{2}=K_{3}$ (3DDMP); $M_{3} s=M_{3} d$ (3D-BBE Volterra); $M_{2}$ (2D P-A Volterra).

Therefore, for example, by varying the values of delay $\mathrm{T} 1$, we are varying the parameter $N_{1}$ in the 3D-DMP model, while by varying delay $\mathrm{T} 2$, we are varying simultaneously the parameters $N_{2}=N_{3}=M_{2}=K_{3}$, as it can be observed in (14)-(16). As it was expected, when considering memory effects these behavioral models can become intractable from the computational complexity point of view. However, the 3DDMP, thanks to its distributed structure, is the one that shows a slower growth in the number of parameters with the number of memory taps considered. In the particular case of the 2D P-A Volterra, the number of coefficients do not experience a significant growth by increasing the memory depth, however, the complexity of the model increases by considering higher order kernels of the 2D P-A Volterra model, as evidenced in Fig. 6. A comparison of the linearization performance of the aforementioned models will be given in Section V.

\section{Further Model Order Reduction Based on the PCA Theory}

To further reduce the model order of the 3D-DMP DPD, we have considered a technique that is based on the principal component analysis (PCA) theory [25], where by converting a basis of observed and eventually correlated data into a basis of uncorrelated data, we can eliminate redundancies and thus reduce the order of the DPD model. Moreover, applying order reduction techniques also improves the conditioning of the basis waveforms used [29].

An example on how to apply the PCA theory to reduce the model order of a DPD was published by the authors in [28]. With this technique we can perform a change of basis where the number of required coefficients decreases by a certain reduction factor (RDF), i.e., \# coeff. of the original basis divided by the \# coeff. of the new basis. The PCA approach relaxes the computational load of the subsystem responsible for assisting the real-time FPGA device in the task of updating the DPD coefficients, such as for example, a soft-core microprocessor (e.g., Xilinx Microblaze) or any 
other microprocessor device. In addition, it prevents from the well-known ill-conditioning identification problem because the resulting new basis is orthogonal. In real-time, the new basis is directly built through a linear combination of the old one. This may increase the DPD computational complexity, unless additional punning strategies are considered. As reported in [28], further reduction of the terms participating in the linear combination that produces the new basis can be performed without significantly degrading the overall DPD performance. As a consequence the PCA approach can be useful not only to improve the data matrix conditioning but also to reduce the whole DPD system computational complexity.

\section{EXPERIMENTAL SETUP}

For the concurrent dual-band transmission with dynamic supply we used a WCDMA signal of $5 \mathrm{MHz} \mathrm{BW}$ at $1.75 \mathrm{GHz}$ and $10 \mathrm{MHz}$ BW LTE signal at $2.1 \mathrm{GHz}$ generated with the pattern generator software from Texas Instruments (TI). The PAPR of the dual-band signal used to test the 3D-DMP DPD was $9.2 \mathrm{~dB}$ measured at $10^{-4}$ of the CCDF. The experimental test bench is illustrated in Fig. 7. The generated waveform passes through the PC and DPD blocks implemented in Matlab and is downloaded for playback in the TI boards (TSW1400EVM pattern generator + TSW30H84EVM DACs and IQ modulator) that output the signal that will be fed into the PA with 16-bit resolution at $614.4 \mathrm{MS} / \mathrm{s}$ after upconversion at the $2 \mathrm{GHz}$ carrier frequency. The wide bandwidth of the TI boards allows the generation of the composite dual-band signals at baseband and thus, avoiding timing misalignment between both concurrent signals at different bands, as is the case of using two signal generators and frequency up-conversion units [12]. However, the price to pay is that the frequency band separation is limited and determined by the maximum DAC sampling frequency. A Tabor WW2572A arbitrary wave generator was used as DAC to output the supply envelope (i.e., the sum of baseband envelopes or a SR reduced version of the original envelope) that was previously generated in Matlab. The DUT was a Cree Inc. Evaluation Board CGH40006P-TB (GaN transistor). A Linear Technology IC LT1210 (35 MHz bandwidth and $900 \mathrm{~V} / \mathrm{s}$ of slew-rate at $\mathrm{Av}=2$ and $10 \Omega$ load) was considered to build the envelope driver. Because we are using a linear but slightly efficient envelope amplifier, the power efficiency values reported in Section V obviate the envelope driver's efficiency and thus, only the drain efficiency of the RF PA is reported. Finally, a digital storage oscilloscope (DSO Agilent 90404A) was used to acquire the RF output signal with 8 bit resolution and a maximum sampling rate of $20 \mathrm{GS} / \mathrm{s}$.

\section{EXPERIMENTAL RESULTS}

In a first approach, the proposed 3D-DMP DPD model is compared with the 3D-MP, 3D-BBE Volterra and 2D P-A Volterra in Table I. The comparison of the linearity performance is evaluated in terms of in-band (i.e., NMSE) and out-of-band (i.e., ACLR) distortion compensation. In addition, the computational complexity is compared in terms of number of coefficients used by the DPD. Without loss of

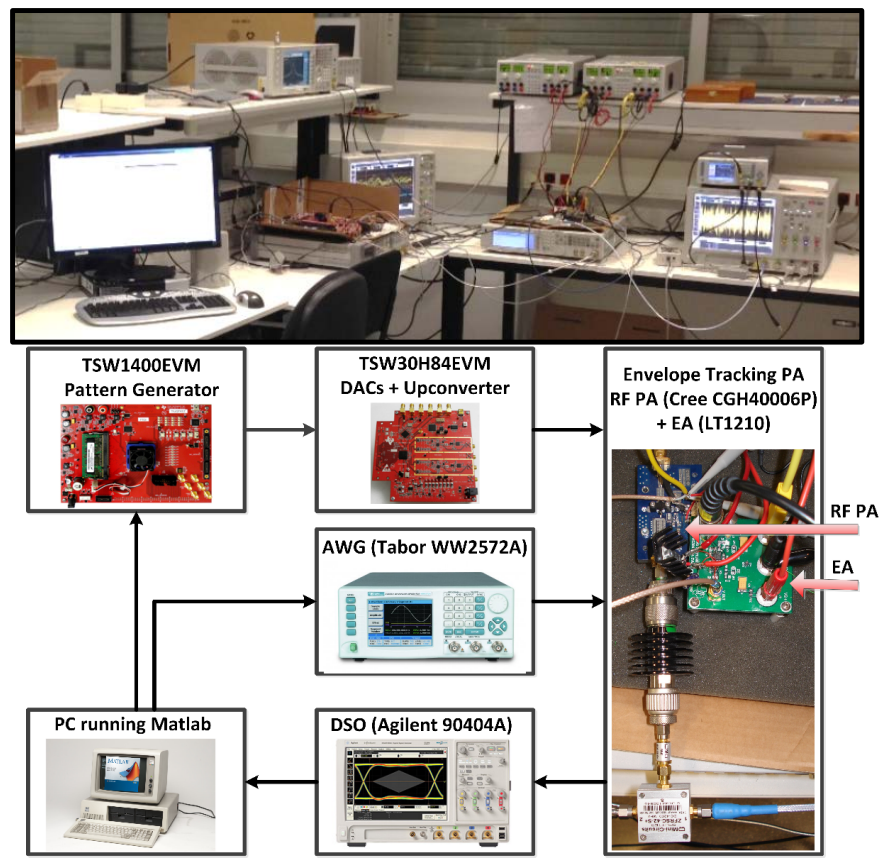

Fig. 7. Experimental test bench.

TABLE I

COMPARISON OF DIFFERENT DUAL-BAND ENVELOPE TRACKING DPD Behavioral Models and Supply Modulation Based on the SR\&BWRED ENVELOPE WITH LTP $=40 \%$.

\begin{tabular}{|c|c|c|c|c|c|}
\hline \multirow{2}{*}{$\begin{array}{l}\text { DB ET DPD } \\
\text { Beh. Models }\end{array}$} & \multicolumn{2}{|c|}{ ACLR (dB) } & \multicolumn{2}{|c|}{ NMSE (dB) } & \multirow{2}{*}{$\begin{array}{l}\text { Num. } \\
\text { coeff. }\end{array}$} \\
\hline & $\begin{array}{l}\text { Band } 1 \\
@ 1.75 \mathrm{GHz}\end{array}$ & $\begin{array}{l}\text { Band } 2 \\
@ 2.1 \mathrm{GHz}\end{array}$ & $\begin{array}{l}\text { Band } 1 \\
\text { wCDMA }\end{array}$ & $\begin{array}{l}\text { Band } 2 \\
\text { LTE-10MHz }\end{array}$ & \\
\hline 3D MP ${ }_{(P=5, Q=3}$, & L: -43.0 & L: -43.5 & -35.0 & -34.1 & 90 \\
\hline$R=3, N=2, M=1$ & U: -42.3 & U: -43.1 & & & \\
\hline \multicolumn{6}{|l|}{ 3D BBE Volterra } \\
\hline$M_{1}=14, M_{3, s, d}=2$ & L: -41.1 & L: -39.3 & -32.5 & -32.4 & 99 \\
\hline$\left.N_{s}=3, M_{5, s, d 1, d 2}=0\right)$ & U: -40.5 & U: -39.1 & & & \\
\hline 2D P-A Volterra & L: -44.1 & L: -42.9 & -34.2 & -33.8 & 128 \\
\hline$M_{1}=1, M_{2}=1, N=5$ & U: -44.0 & U: -42.5 & & & \\
\hline \multicolumn{6}{|l|}{ 3D DMP $\left(P_{1}=7, R_{3}=3\right.$, } \\
\hline$P_{2}=P_{3}=Q_{2}=5, N_{1}=6$, & L: -46.6 & L: -44.1 & -34.6 & -36.7 & 97 \\
\hline$\left.N_{2}=N_{3}=M_{2}=1, K_{3}=2\right)$ & U: -46.4 & U: -43.8 & & & \\
\hline 3D DMP & L: -47.4 & L: -45.5 & -36.5 & -37.6 & 96 \\
\hline 482 coeff w. $R D F=5$ & $\mathrm{U}:-47.3$ & U: -45.5 & & & \\
\hline
\end{tabular}

generality we can assume that a higher number of coefficients implies higher computational load and thus higher power consumption of the overall digital signal processing system. In Table I, we have considered a fixed number of parameters (around 100 coefficients) in order to see the linearization performance when considering a dynamic supply based on the SR\&BWred envelope and with LTP $=40 \%$. It can be observed that, thanks to the distributed structure of the 3DDMP DPD, we have more flexibility (degrees of freedom or resolution) to determine the most accurate configuration when only considering $\sim 100$ coefficients. As a consequence, despite the rest of the behavioral models under comparison can perform equally well when considering a higher number of coefficients, the best linearization performance taking into 
TABLE II

3D Digital Predistortion with Model Order Reduction and Different Supply Modulation TechniQues.

\begin{tabular}{|c|c|c|c|c|c|c|c|}
\hline \multirow{2}{*}{$\begin{array}{l}\text { Digital Predistortion } \\
\text { 3D Model }\end{array}$} & \multirow{2}{*}{$\begin{array}{l}\text { Supply Modulation } \\
\text { Technique }\end{array}$} & \multirow[b]{2}{*}{ Reduction Factor } & \multicolumn{2}{|c|}{ ACLR (dB) } & \multicolumn{2}{|c|}{ NMSE (dB) } & \multirow{2}{*}{$\begin{array}{l}\text { Num. } \\
\text { coeff. }\end{array}$} \\
\hline & & & $\begin{array}{l}\text { Band } 1 \\
@ 1.75 \mathrm{GHz}\end{array}$ & $\begin{array}{l}\text { Band } 2 \\
@ 2.1 \mathrm{GHz}\end{array}$ & $\begin{array}{c}\text { Band } 1 \\
\text { wCDMA }\end{array}$ & $\begin{array}{l}\text { Band } 2 \\
\text { LTE-10MHz }\end{array}$ & \\
\hline \multirow{6}{*}{$\begin{array}{l}\text { 3D-Memory Polynomial } \\
(P=7, Q=5, R=3, N=6, M=4)\end{array}$} & \multirow{6}{*}{ SR\&BWred Env. } & \multirow[t]{2}{*}{$R D F=1$} & L: -49.0 & L: -47.5 & -37.1 & -36.9 & \multirow[t]{2}{*}{2520} \\
\hline & & & $\mathrm{U}:-49.3$ & $\mathrm{U}:-47.5$ & & & \\
\hline & & \multirow[t]{2}{*}{$R D F=8$} & L: -45.7 & L: -45.3 & -34.2 & -36.7 & \multirow[t]{2}{*}{315} \\
\hline & & & U: -46.0 & $\mathrm{U}:-45.1$ & & & \\
\hline & & \multirow[t]{2}{*}{$R D F=64$} & L: -42.3 & L: -41.2 & -32.3 & -33.7 & \multirow[t]{2}{*}{39} \\
\hline & & & U: -42.1 & $\mathrm{U}:-41.3$ & & & \\
\hline \multirow{12}{*}{$\begin{array}{l}\text { 3D-Distributed Memory Polynomial } \\
\qquad \begin{array}{l}P_{1}=7, P_{2}=P_{3}=Q_{2}=5, N_{1}=10 \\
\left.R_{3}=3, N_{2}=N_{3}=M_{2}=3, K_{3}=4\right)\end{array}\end{array}$} & \multirow{6}{*}{ SR\&BWred Env. } & \multirow[t]{2}{*}{$R D F=1$} & L: -48.6 & L: -48.5 & -36.3 & -36.0 & \multirow[t]{2}{*}{482} \\
\hline & & & U: -49.0 & U: -48.6 & & & \\
\hline & & \multirow[t]{2}{*}{$R D F=5$} & L: -47.4 & L: -45.5 & -36.5 & -37.6 & \multirow[t]{2}{*}{96} \\
\hline & & & U: -47.3 & $\mathrm{U}:-45.5$ & & & \\
\hline & & \multirow[t]{2}{*}{$R D F=16$} & L: -40.0 & L: -40.6 & -32.7 & -33.2 & \multirow[t]{2}{*}{30} \\
\hline & & & U: -40.0 & U: -40.5 & & & \\
\hline & \multirow{6}{*}{ AVG (GM p=2) Env. } & \multirow[t]{2}{*}{$R D F=1$} & L: -49.7 & L: -48.6 & -36.5 & -39.2 & \multirow[t]{2}{*}{482} \\
\hline & & & U: -49.8 & $\mathrm{U}:-48.8$ & & & \\
\hline & & \multirow[t]{2}{*}{$R D F=9$} & L: -46.8 & L: -45.4 & -36.5 & -34.2 & \multirow[t]{2}{*}{54} \\
\hline & & & U: -45.8 & U: -45.2 & & & \\
\hline & & \multirow[t]{2}{*}{$R D F=16$} & L: -43.1 & L: -42.5 & -34.9 & -34.3 & \multirow[t]{2}{*}{30} \\
\hline & & & U: -42.8 & $\mathrm{U}:-42.0$ & & & \\
\hline
\end{tabular}

account only $\sim 100$ coefficients is achieved with the 3D-DMP DPD. In addition, if instead of empirically finding the most accurate configuration (polynomial order, number of kernels, memory depth, etc.) we start from an initial configuration with a lot of coefficients and then we perform model order reduction based on the PCA theory, we can observe in Table I (last row) that it is possible to improve the aforementioned 3D-DMP DPD linearity figures. Therefore, the linearity requirements for the LTE signal (i.e., $-45 \mathrm{dBc}$ of ACLR) cannot be simply met (with this particular restriction: $\sim 100$ coefficients and supply modulation based on the SR\&BWred Envelope with LTP $=40 \%$ ) unless we consider a 3D-DMP DPD with 482 coefficients and then we apply a reduction factor (RDF) of $R D F=5$, resulting in $\operatorname{round}\left(\frac{482}{R D F}\right)=96$ coefficients.

Table II show the linearization performance of the 3DMP DPD and the proposed 3D-DMP DPD, respectively, for different RDFs and considering dynamic supply modulation based on the SR\&BWred envelope with LTP $=40 \%$ and on the AVG (GM $p=2$ ) envelope with LTP $=30 \%$. With the PCA technique, we can reduce the number of coefficients several factors before significantly degrading both in-band and outof-band linearity figures. Therefore, for example, taking into account the $-45 \mathrm{dBc}$ ACLR restriction for the LTE signal, with the 3D-MP DPD (see Table II) it is possible to apply a reduction up to $R D F=8$ (315 coefficients) and still be compliant with the spectrum mask. Again, taking advantage of the distributed structure of the proposed 3D-DMP (see Table II), this time with only 96 coefficients $(R D F=5)$ we can already meet the linearity specifications in Band 2. Moreover, by using dynamic supply modulation based on the AVG envelope we obtain better linearity figures and, for example, we can meet the $-45 \mathrm{dBc}$ ACLR specification with the 3DDMP DPD considering only 54 coefficients $(R D F=9)$.

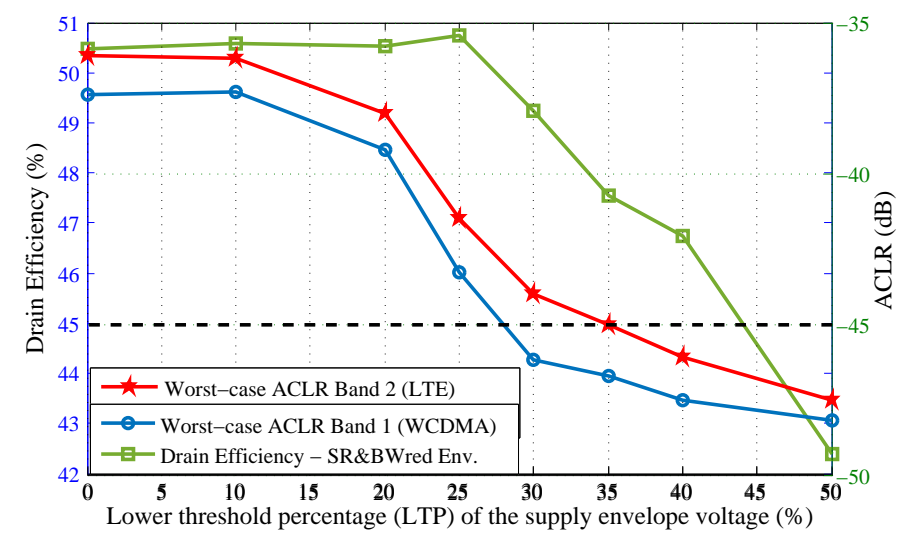

Fig. 8. Drain efficiency and worst-case ALCR for different LTP and considering 3D-DMP DPD and supply modulation based on the SR\&BWred envelope.

Fig. 8 and Fig. 9 show the measured drain efficiency and the worst-case ACLR (after applying 3D-DMP DPD) for different LTP values when considering supply modulations based on the SR\&BWred and AVG envelopes, respectively. As it was expected, the more DC power the PA consumes (i.e., higher LTP values) the better ACLR figures and the worse drain efficiency. From the power efficiency perspective, we would like to keep the LTP to the minimum but, to be compliant with the desired ACLR levels the LTP has to be at least $L T P=33 \%$ in the case of supplying with the SR\&BWred envelope and $L T P=23 \%$ in the case of using the AVG envelope.

Table III shows a comparison of the drain efficiency (DE) obtained with different envelope tracking supply modulation techniques: based on the GM of the instantaneous dual-band 
TABLE III

EFFICIENCY IMPROVEMENT FOR DIFFERENT SUPPLy MODULATION TECHNIQUES IN DUAL-BAND ET PAs WITH 3D-DMP DPD.

\begin{tabular}{|c|c|c|c|c|c|c|c|}
\hline \multirow[b]{2}{*}{ Configuration } & \multicolumn{2}{|c|}{ ACLR (dB) } & \multicolumn{2}{|c|}{ NMSE (dB) } & \multirow{2}{*}{$\begin{array}{c}\text { Mean } \\
\text { Output } \\
\text { Power }(\mathrm{dBm})\end{array}$} & \multirow[b]{2}{*}{$\begin{array}{l}\mathrm{DE} \\
(\%)\end{array}$} & \multirow[b]{2}{*}{ EI } \\
\hline & $\begin{array}{c}\text { Band } 1 \\
@ 1.75 \mathrm{GHz}\end{array}$ & $\begin{array}{c}\text { Band } 2 \\
\text { @2.1 GHz }\end{array}$ & $\begin{array}{l}\text { Band } 1 \\
\text { WCDMA }\end{array}$ & $\begin{array}{c}\text { Band } 2 \\
\text { LTE-10 MHz }\end{array}$ & & & \\
\hline Fixed Supply & L: -41.0 & L: -43.6 & -31.2 & -32.5 & 28.0 & 26.3 & - \\
\hline No DPD & U: -38.0 & U: -40.3 & & & & & \\
\hline $\begin{array}{l}\text { Fixed Supply } \\
\text { with 2D-DPD. }\end{array}$ & $\begin{array}{l}\text { L: }-51.3 \\
\text { U: }-51.1\end{array}$ & $\begin{array}{l}\text { L: }-50.9 \\
\text { U: }-51.1\end{array}$ & -35.2 & -36.3 & 28.1 & 26.8 & 1 \\
\hline $\begin{array}{l}\text { Supply Mod.:GM p=1 (PEAK) Env. LTP=33\% } \\
\text { No DPD }\end{array}$ & $\begin{array}{l}\mathrm{L}:-24.2 \\
\mathrm{U}:-24.0\end{array}$ & $\begin{array}{l}\mathrm{L}:-25.4 \\
\mathrm{U}:-25.3\end{array}$ & -15.5 & -16.0 & 28.1 & 50.0 & - \\
\hline $\begin{array}{l}\text { Supply Mod.:GM } p=1 \text { (PEAK) Env. LTP=33\% } \\
\text { with 3D-DMP DPD }\end{array}$ & $\begin{array}{l}\text { L: }-47.2 \\
\text { U: }-46.7\end{array}$ & $\begin{array}{l}\text { L: }-45.7 \\
\text { U: }-45.6\end{array}$ & -35.9 & -36.8 & 28.0 & 47.4 & 1.77 \\
\hline $\begin{array}{l}\text { Supply Mod.:GM } p=1.5 \text { Env. } L T P=25 \% \\
\text { No DPD }\end{array}$ & $\begin{array}{l}\text { L: }-24.2 \\
\text { U: }-24.0\end{array}$ & $\begin{array}{l}\text { L: }-24.7 \\
\text { U: }-24.6\end{array}$ & -15.8 & -15.3 & 28.1 & 50.6 & - \\
\hline $\begin{array}{l}\text { Supply Mod.: } G M p=1.5 \text { Env. } L T P=25 \% \\
\text { with 3D-DMP DPD }\end{array}$ & $\begin{array}{l}\text { L: }-46.4 \\
\text { U: }-46.0\end{array}$ & $\begin{array}{l}\text { L: }-45.3 \\
\text { U: }-45.2\end{array}$ & -36.2 & -36.8 & 28.0 & 48.0 & 1.79 \\
\hline $\begin{array}{l}\text { Supply Mod.:GM } p=2(A V G) \text { Env. LTP }=23 \% \\
\text { No DPD }\end{array}$ & $\begin{array}{l}\text { L: }-24.3 \\
\text { U: }-24.2\end{array}$ & $\begin{array}{l}\text { L: }-24.3 \\
\text { U: }-24.2\end{array}$ & -15.8 & -15.2 & 28.2 & 50.2 & - \\
\hline $\begin{array}{l}\text { Supply Mod.:GM } p=2(A V G) \text { Env. LTP }=23 \% \\
\text { with 3D-DMP DPD }\end{array}$ & $\begin{array}{l}\text { L: }-47.7 \\
\text { U: }-47.6\end{array}$ & $\begin{array}{l}\text { L: }-46.1 \\
\text { U: }-46.0\end{array}$ & -35.4 & -35.4 & 28.0 & 47.5 & 1.77 \\
\hline $\begin{array}{l}\text { Supply Mod.: GM } p=4 \text { Env. } L T P=23 \% \\
\text { No DPD }\end{array}$ & $\begin{array}{l}\text { L: }-25.9 \\
\text { U: }-25.8\end{array}$ & $\begin{array}{l}\text { L: }-24.7 \\
\text { U: }-24.6\end{array}$ & -17.3 & -16.0 & 28.0 & 49.8 & - \\
\hline $\begin{array}{l}\text { Supply Mod.:GM } p=4 \text { Env. } L T P=23 \% \\
\text { with 3D-DMP DPD }\end{array}$ & $\begin{array}{l}\text { L: }-48.1 \\
\text { U: }-47.6\end{array}$ & $\begin{array}{l}\text { L: }-45.6 \\
\text { U: }-45.6\end{array}$ & -35.2 & -37.9 & 28.0 & 47.6 & 1.78 \\
\hline $\begin{array}{l}\text { Supply Mod.:SR\&BWred Env. } L T P=33 \% \\
\text { No DPD }\end{array}$ & $\begin{array}{l}\text { L: }-24.6 \\
\text { U: }-24.4\end{array}$ & $\begin{array}{l}\text { L: }-25.8 \\
\text { U: }-25.7\end{array}$ & -16.2 & -15.8 & 28.1 & 51.5 & - \\
\hline $\begin{array}{l}\text { Supply Mod.:SR\&BWred Env. LTP=33\% } \\
\text { with 3D-DMP DPD }\end{array}$ & $\begin{array}{l}\text { L: }-47.0 \\
\text { U: }-46.9\end{array}$ & $\begin{array}{l}\text { L: }-45.4 \\
\text { U: }-45.3\end{array}$ & -36.0 & -36.0 & 28.0 & 48.8 & 1.82 \\
\hline
\end{tabular}

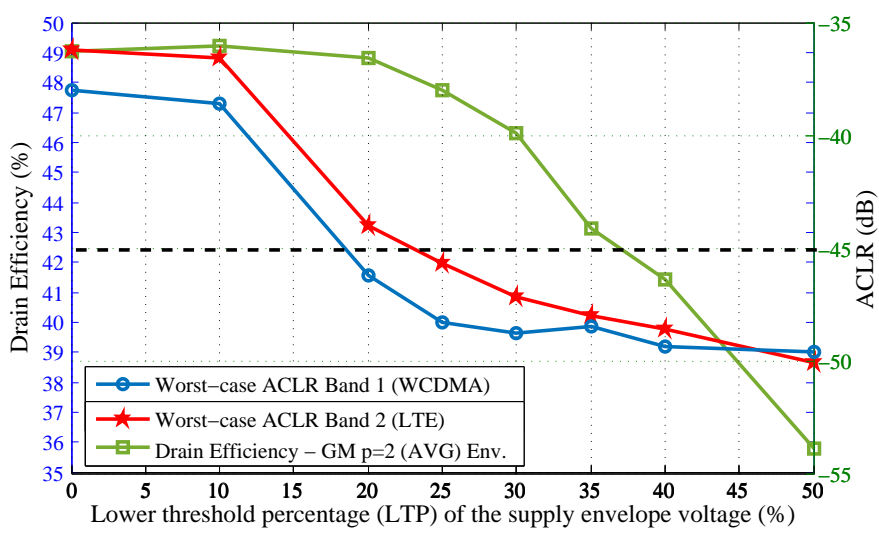

Fig. 9. Drain efficiency and worst-case ALCR for different LTP and considering 3D-DMP DPD and supply modulation based on the AVG envelope.

envelope for $p=[1,1.5,2,4]$, and based on the SR\&BWred envelope of the instantaneous dual-band envelope. In all cases under comparison, the ACLR values after 3D-DMP DPD are below $-45 \mathrm{~dB}$, while the mean output power is $28 \mathrm{dBm}$. The 3D-DMP DPD used 121 coefficients after applying PCA with $R D F=4$. A straightforward way to compare the drain efficiency of each configuration is through the efficiency improvement (EI) metric [19], defined as the ratio between the fixed $\left(P_{F S}\right)$ and the dynamic supply $\left(P_{D S}\right)$ power consumption, $E I=\frac{P_{F S}}{P_{D S}}$. As a consequence, the higher the
EI the better. As shown in Table III, there is no significant differences in the EI among the different supply strategies. However, it is expected that by decreasing the SR reduction percentage (currently at $98.87 \%$ ) and thus having a faster supply modulated envelope, the EI of the SR\&BW strategy would be significantly better than the ones based on the GM. Unfortunately, we are currently limited in SR by the linear supply modulator described in Section IV.

To evidence the 3D-DMP DPD linearization performance, Fig. 10 and Fig. 11 show the output spectra and the gain characteristics respectively, before and after linearization at both frequency bands and considering the SR\&BWred envelope to dynamically supply the PA. Similarly, Fig. 12 and Fig. 13 show the output spectra and the gain characteristics but this time considering the AVG envelope to dynamically supply the PA. The typical slow-envelope dependent unwanted distortion effects can be observed in these plots of the gain.

\section{CONCLUSION}

In this paper we have presented a new DPD for concurrent dual-band envelope tracking PAs. The advantage of the proposed 3D-DMP DPD in comparison to the already published solutions is that, thanks to its distributed structure in which several cross-products among the three input variables are obviated, the number of coefficients required to characterize and compensate for the in-band, out-of-band, cross-band inter- 

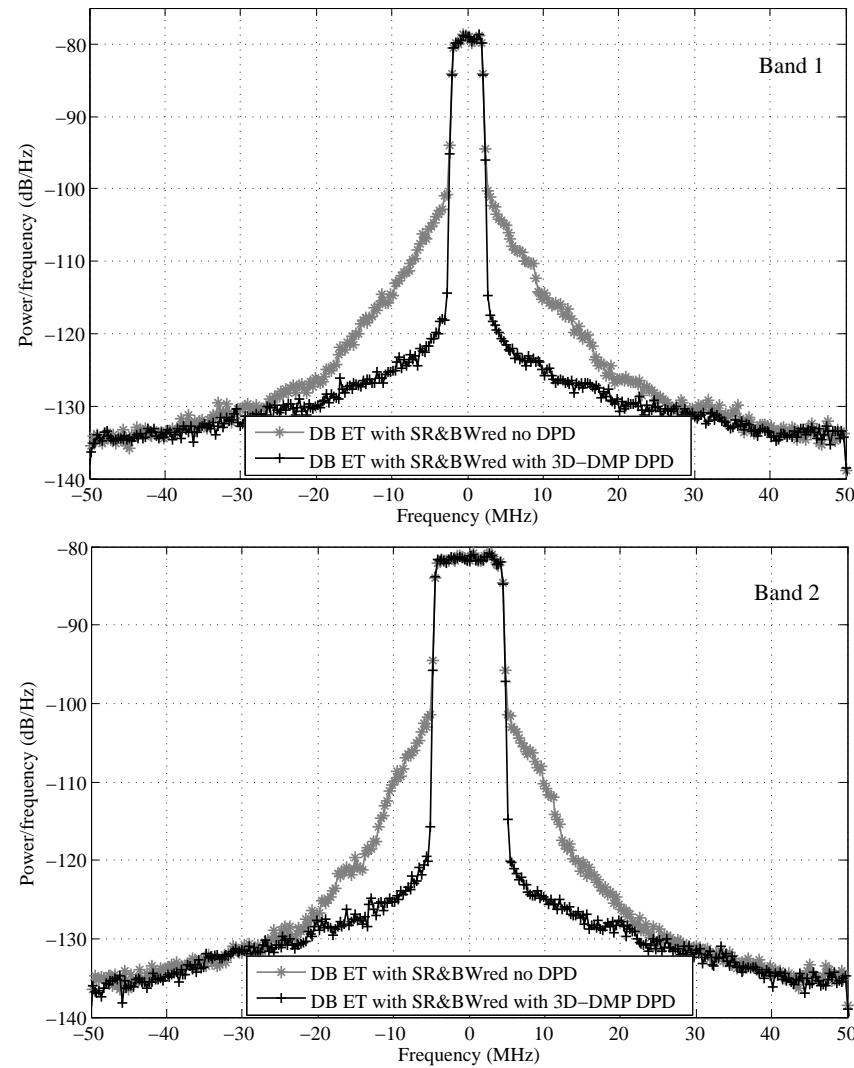

Fig. 10. Linearized (with 3D-DMP DPD) and unlinearized output power spectra of a WCDMA (upper) and LTE (lower) signals. The PA is dynamically supplied with the SR\&BWred envelope.
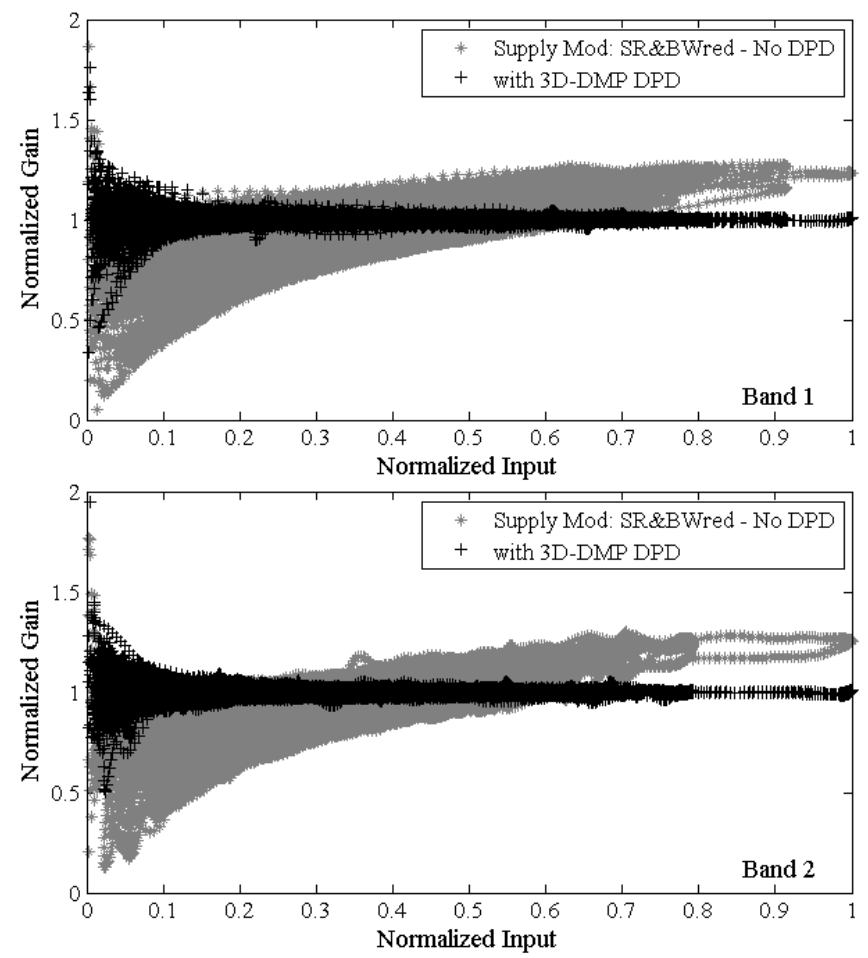

Fig. 11. Normalized Gain characteristics before and after 3D-DMP DPD with supply modulation based on the SR\&BWred envelope
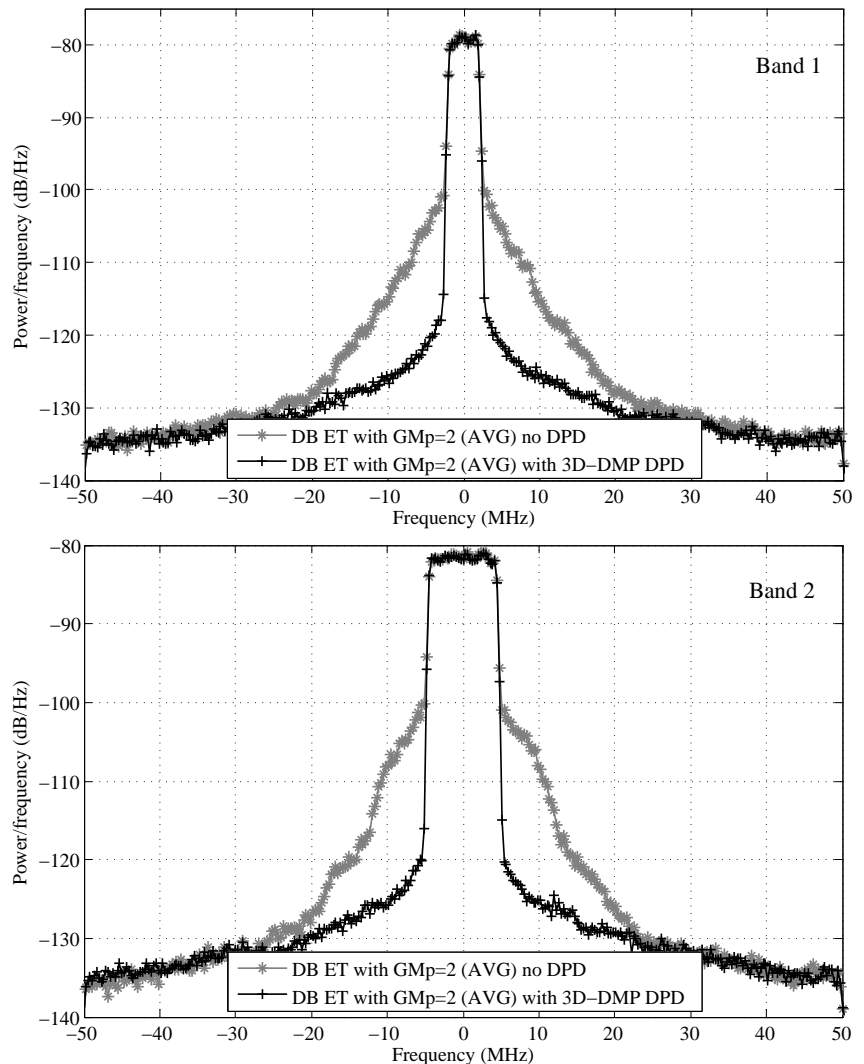

Fig. 12. Linearized (with 3D-DMP DPD) and unlinearized output power spectra of a WCDMA (upper) and LTE (lower) signals. The PA is dynamically supplied with the AVG envelope.
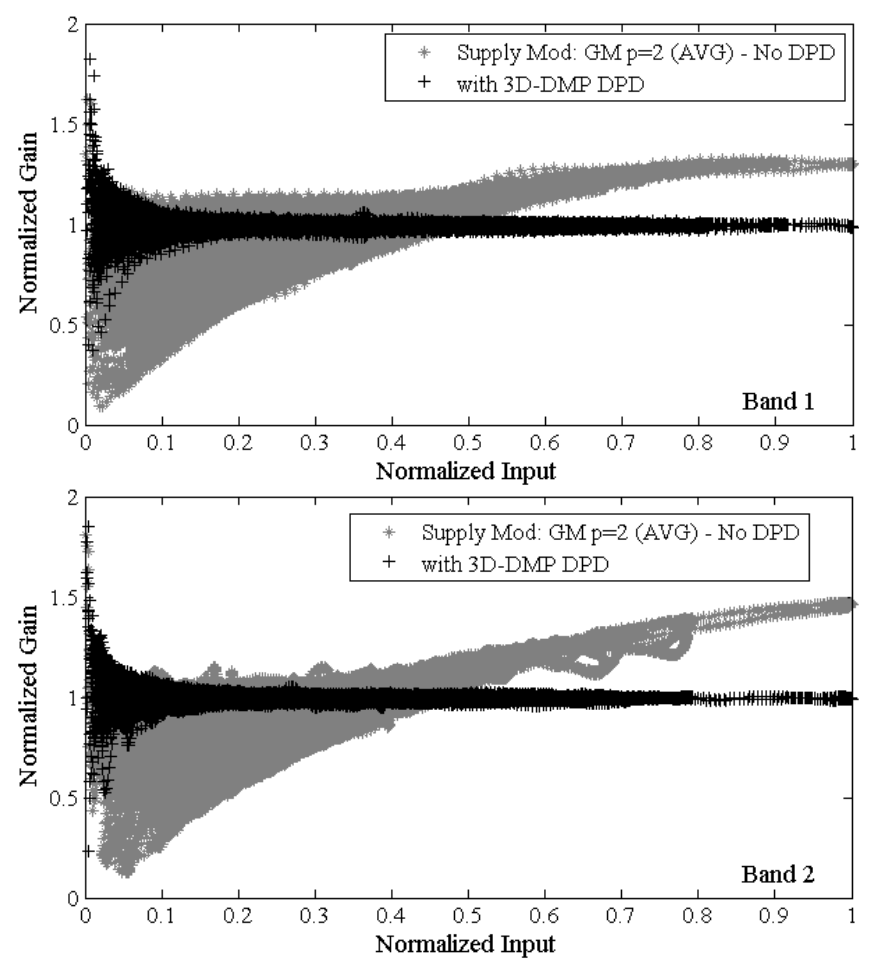

Fig. 13. Normalized Gain characteristics before and after 3D-DMP DPD with supply modulation based on the AVG envelope 
modulation and slow-envelope dependent distortion is significantly lower than in its Volterra-based or 3D-MP counterparts. Moreover, with the model order reduction technique based on the PCA theory we can determine the minimum number of coefficients required to meet the targeted ACLR values.

The proposed 3D-DMP DPD has been tested under different envelope tracking supply modulation techniques. The reported EI figures are quite similar among the different strategies considered in this paper. However, it is expected that diminishing the SR reduction percentage, better EI figures could be achieved using the SR\&BWred envelope. Unfortunately, this cannot be proved due to the SR \& BW limitations of the envelope modulator used in this paper. Instead, the best linearability is found when using the AVG envelope (GM $p=2$ ), since it is the supply modulation technique presenting the narrowest BW.

We can conclude that, from the power efficiency perspective, the slow envelope should be as fast as supported by the envelope driver, i.e., operating with the minimum necessary SRred percentage. From the computational complexity point of view, we want to meet the ACLR specifications with the minimum number of coefficients (maximum RDF when using PCA-based model order reduction). Finally, the LTP of the supply envelope voltage has shown to be a key parameter to cope with the linearity versus efficiency trade-off.

\section{ACKNOWLEDGMENT}

The authors would like to thank Dr. José Angel García from Universidad de Cantabria for his useful comments and interesting discussions on concurrent dual-band envelope tracking power amplifiers.

\section{REFERENCES}

[1] A. Cidronali, N. Giovannelli, T. Vlasits, R. Hernaman, and G. Manes, "A 240W dual-band 870 and $2140 \mathrm{MHz}$ Envelope Tracking GaN PA designed by a probability distribution conscious approach," in IEEE MTT-S International Microwave Symposium Digest (MTT), 2011, pp. $1-4$.

[2] P. Saad, P. Colantonio, L. Piazzon, F. Giannini, K. Andersson, and C. Fager, "Design of a Concurrent Dual-Band $1.8-2.4-\mathrm{GHz}$ GaNHEMT Doherty Power Amplifier," IEEE Transactions on Microwave Theory and Techniques, vol. 60, no. 6, pp. 1840-1849, 2012.

[3] W. Chen, S. Zhang, Y. Liu, Y. Liu, and F. Ghannouchi, "A concurrent dual-band uneven doherty power amplifier with frequency-dependent input power division," IEEE Transactions on Circuits and Systems I: Regular Papers, vol. 61, no. 2, pp. 552-561, Feb 2014.

[4] X. Fu, D. Bespalko, and S. Boumaiza, "Novel dual-band matching network for effective design of concurrent dual-band power amplifiers," IEEE Transactions on Circuits and Systems I: Regular Papers, vol. 61, no. 1, pp. 293-301, Jan 2014.

[5] X. Yu and H. Jiang, "Digital predistortion using adaptive basis functions," IEEE Transactions on Circuits and Systems I: Regular Papers, vol. 60, no. 12, pp. 3317-3327, Dec 2013.

[6] Y. Li, J. Lopez, D.-C. Lie, K. Chen, S. Wu, T.-Y. Yang, and G.K. Ma, "Circuits and System Design of RF Polar Transmitters Using Envelope-Tracking and SiGe Power Amplifiers for Mobile WiMAX," IEEE Transactions on Circuits and Systems I: Regular Papers, vol. 58, no. 5, pp. 893-901, May 2011.

[7] N. Giovannelli, A. Cidronali, M. Mercanti, R. Hernaman, G. Wimpenny, and G. Manes, "A $80 \mathrm{~W}$ broadband GaN HEMT envelope tracking PA harmonically tuned for WCDMA and LTE with 50\% average efficiency," in IEEE MTT-S International Microwave Symposium Digest (MTT), 2012, pp. 1-3.
[8] P. L. Gilabert, G. Montoro, D. Lopez, and J. A. Garcia, "3D Digital Predistortion for Dual-Band Envelope Tracking Power Amplifiers," in Asia-Pacific Microwave Conference Proceedings (APMC), 2013, pp. 734-736.

[9] Y. Lin, H. Jang, C. Quindroit, N. Naraharisetti, and P. Roblin, "New Supply Modulation Optimization Methodology for Concurrent Dual Band Envelope Tracking Power Amplifier," in IEEE Wireless and Microwave Technology Conference (WAMICON), June 2014, pp. 1-4.

[10] H. Sarbishaei, B. Fehri, Y. Hu, and S. Boumaiza, "Dual-Band Volterra Series Digital Pre-Distortion for Envelope Tracking Power Amplifiers," IEEE Microwave and Wireless Components Letters, vol. 24, no. 6, pp. 430-432, June 2014.

[11] A. Kwan, M. Younes, S. Zhang, W. Chen, R. Darraji, M. Helaoui, and F. M. Ghannouchi, "Dual-band Predistortion Linearization of an Envelope Modulated Power Amplifier Operated in Concurrent MultiStandard Mode," in IEEE MTT-S International Microwave Symposium Digest (IMS), June 2014.

[12] S. Bassam, M. Helaoui, and F. Ghannouchi, "2-D Digital Predistortion (2-D-DPD) Architecture for Concurrent Dual-Band Transmitters," IEEE Transactions on Microwave Theory and Techniques, vol. 59, no. 10, pp. 2547-2553, 2011.

[13] M. Younes and F. Ghannouchi, "On the modeling and linearization of a concurrent dual-band transmitter exhibiting nonlinear distortion and hardware impairments," IEEE Transactions on Circuits and Systems I: Regular Papers, vol. 60, no. 11, pp. 3055-3068, Nov 2013.

[14] Y.-J. Liu, W. Chen, J. Zhou, B.-H. Zhou, and F. Ghannouchi, "Digital Predistortion for Concurrent Dual-Band Transmitters Using 2-D Modified Memory Polynomials," IEEE Transactions on Microwave Theory and Techniques, vol. 61, no. 1, pp. 281-290, 2013.

[15] N. Naraharisetti, P. Roblin, C. Quindroit, and G. S. Rawat, M., “2D Quasi Exact Inverse of PA model in Digital Predistorter for Concurrent Dual-Band System," in IEEE MTT-S International Microwave Symposium Digest (IMS), 2014.

[16] L. Ding, Z. Yang, and H. Gandhi, "Concurrent Dual-Bandand Digital Predistortion," in IEEE MTT-S International Microwave Symposium Digest (MTT), 2012, pp. 1-3.

[17] A. Kwan, S. Bassam, M. Helaoui, and F. Ghannouchi, "Concurrent dual band digital predistortion using look up tables with variable depths," in IEEE Topical Conference on Power Amplifiers for Wireless and Radio Applications (PAWR), 2013, pp. 25-27.

[18] J. Jeong, D. Kimball, M. Kwak, C. Hsia, P. Draxler, and P. Asbeck, "Wideband Envelope Tracking Power Amplifiers With Reduced Bandwidth Power Supply Waveforms and Adaptive Digital Predistortion Techniques," IEEE Transactions on Microwave Theory and Techniques, vol. 57, no. 12, pp. 3307-3314, 2009.

[19] G. Montoro, P. Gilabert, E. Bertran, and J. Berenguer, "A method for real-time generation of slew-rate limited envelopes in envelope tracking transmitters," in IEEE International Microwave Workshop Series on RF Front-ends for Software Defined and Cognitive Radio Solutions (IMWS), 2010, pp. 1-4.

[20] L. Guan and A. Zhu, "Optimized Low-Complexity Implementation of Least Squares Based Model Extraction for Digital Predistortion of RF Power Amplifiers," IEEE Transactions on Microwave Theory and Techniques, vol. 60, no. 3, pp. 594-603, 2012.

[21] Q. Zhang and Z. Zeng, "A new adaptive algorithm for digital predistortion using LS with singular value decomposition," in International Conference on Information Science and Technology Application (ICISTA), 2013, pp. 169-172.

[22] E. Abd-Elrady, "A recursive prediction error algorithm for digital predistortion of fir wiener systems," in International Symposium on Coтmunication Systems, Networks and Digital Signal Processing (CNSDSP), July 2008, pp. 698-701.

[23] R. Braithwaite, "Wide Bandwidth Adaptive Digital Predistortion of Power Amplifiers Using Reduced Order Memory Correction," in IEEE MTT-S International Microwave Symposium Digest, 2008, pp. 15171520.

[24] M. Rawat, F. Ghannouchi, and K. Rawat, "Three-layered biased memory polynomial for dynamic modeling and predistortion of transmitters with memory," IEEE Transactions on Circuits and Systems I: Regular Papers, vol. 60, no. 3, pp. 768-777, March 2013.

[25] I. Jolliffe, Principal Component Analysis. Springer-Verlag, 2002.

[26] P. Gilabert, G. Montoro, and P. Vizarreta, "Slew-Rate and Efficiency Trade-off in Slow Envelope Tracking Power Amplifiers," in The 7th German Microwave Conference (GeMiC), March 2012, pp. 1-4.

[27] P. Roblin, C. Quindroit, N. Naraharisetti, S. Gheitanchi, and M. Fitton, "Concurrent linearization: The state of the art for modeling and lin- 
earization of multiband power amplifiers," IEEE Microwave Magazine, vol. 14, no. 7, pp. 75-91, Nov 2013.

[28] P. L. Gilabert, G. Montoro, D. Lopez, N. Bartzoudis, E. Bertran, M. Payaro, and A. Hourtane, "Order Reduction of Wideband Digital Predistorters Using Principal Component Analysis," in IEEE MTT-S International Microwave Symposium Digest (IMS), 2013, pp. 1-4.

[29] R. N. Braithwaite, Digital Processing for Front End in Wireless Communication and Broadcasting. Cambridge University Press, 2011, ch. General principles and design overview of digital predistortion, pp. 143191.

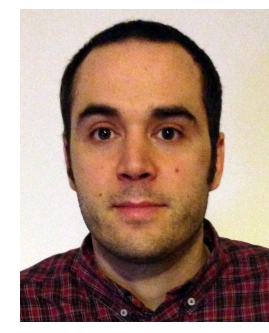

Pere L. Gilabert (M'09-SM'13) received his M.Sc. degree in Telecommunication Engineering from the Universitat Politècnica de Catalunya (UPC) in 2002, and he developed his Master Thesis at the University of Rome La Sapienza with an Erasmus exchange grant. He joined the department of Signal Theory and Communications (TSC) in 2003 and received his $\mathrm{Ph} . \mathrm{D}$., awarded with the Extraordinary Doctoral Prize, from the UPC in 2008. He is an Associate Professor at the Castelldefels School of Telecommunications and Aerospace Engineering (EETACUPC). His research activity is in the field of linearization techniques and digital signal processing solutions for highly efficient transmitter architectures.

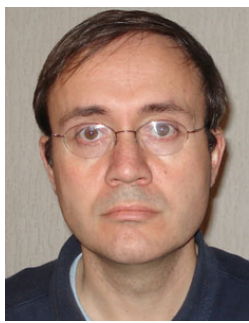

Gabriel Montoro (M'09) received the M.Sc. degree in Telecommunication Engineering in 1990 and his Ph.D. degree in 1996, both from the Universitat Politècnica de Catalunya (UPC). He joined the department of Signal Theory and Communications (TSC) in 1991, where he is currently an Associate Professor. His first research works were done on the area of adaptive control, and now his main research interest is in the use of signal processing strategies for power efficiency improvement in communications systems. 